\title{
Performance Analysis in Wireless Home Network using Bluetooth with SAW-ARQ
}

\author{
Sung-Hwa Hong ${ }^{1^{*}}$ and Bong-Jik Kang ${ }^{1}$ \\ ${ }^{1}$ Department of Software Engineering, Dongyang Mirae University \\ $\mathrm{SAW}-\mathrm{ARQ}$ 를 활용하여 블루투스 하에서의 무선 홈 네트워크의 \\ 성능 분석 \\ 홍성화 $^{1^{*},}$, 강봉직 ${ }^{1}$ \\ ${ }^{1}$ 동양미래대학 소프트웨어정보과
}

\begin{abstract}
In this paper, we proposed the method called "DoublePico"(Double Piconet) forovercoming low data transmission rate in a scatternet. This needs a new Ad-hoc network topology to transmit with high rate. Every node performs the function of the relay station. DoublePico has two bluetooth devices that should form two different poconets; every node can make a link between two different piconets. Two different piconets are linked in one node by the link with two bluetooth divices thereby forming the Ad-Hoc network. In this paper, we shows the method of DoublePico which supports about $457 \mathrm{kbps}$ of the maximum data transmission rate. This method supports a higher data transmission rate than the traditional bluetooth's Ad-Hoc topology by using analysis and comparison of existing algorithm of bluetooth specification and simulation results. Specifically, this paper focuses on the impact of intererence on the PER (Packet Error Rate), throughput performance, and the throughput improvement with SAW(Stop and Wait)-ARQ scheme in DoublePico.
\end{abstract}

요 약 본 논문에서, 우리는 스캐터넷에서 저속 데이터 전송을 극복하기 위한 더블피코(Double Pico) 방안을 제서히 였다. 이는 고속 전송을 위한 새로운 애드혹 네트워크 토폴로지를 필요로 한다. 모든 노드는 릴레이 장비를 위해 기 능을 동작한다. 더블 피코는 서로 다른 피코넷을 형성하는 두 블루투스 장비를 가지고 있다; 모든 노드는 서로 다른 피코넷에서 링크를 만들 수 있다. 서로 다른 두 피코넷은 두 개의 블루투스 장비의 링크에의해 하나의 노드로 링크되 며, 따라서 애드혹 망을 구성하게 된다. 본 논문에서는 최대 전송 속도 $475 \mathrm{kbps}$ 를 지원하는 더블 피코 방안을 제시하 였다. 이 방안은 일반적인 블루투스 스펙과 시뮬레이션 결과와 비교하여 전통적인 블루투스 애드혹 토폴로지보다 보 다 빠른 속도를 지원한다. 특히 본 논문에서는 PER에서의 간섭 효과, 성능 분석과 더블 피코하에서의 SAW(Stop and Wait)-ARQ 성능 향상에 초점을 두었다.

Key Words : Home Network, Multi-Hop, Bluetooth, Routing, SAW-ARQ

\section{Introduction}

The increasing reliance on information available on Internet, and the rapid growth of the wireless subscriber population suggest a need for Internet users to maintain communications as they move from place to place, the popularity of portable computers combined with the growth of wireless networks and services has led to many efforts to make mobile computing an every reality. To achieve this goal, designers need information about the behavior of mobile hosts (portable computers) and the characteristics of the networks they use. Therefore, solutions have been proposed to support mobility in the future IP based wireless networks [1,2].

${ }^{*}$ Corresponding Author : Hong, Sung-Hwa(shhong@dongyang.ac.kr)

Received November 10, 2009

Revised (1st January 22, 2010, 2nd March 8, 2010)

Accepted May 13, 2010 
The latest advances in the Internet technologies, the dropping of PC rates, ad the proliferation of smart devices in the house, have dramatically increased the need for an efficient home network. A home network connects several computers and networked appliances within a house using Ethernet or wireless technology, and then connects them all to the Internet through the home gateway. Caching at the home Internet gateway is an effective solution to improve the performance of the home network.

Home networking deals with connecting multimedia devices and facilitating storage, retrieval and viewing digital media on these devices. Commonly, distribution of entertainment content between devices is done via dedicated wired interfaces. However, wireless communication would offer the end user enhanced benefits of easy connectivity and mobility in the home. The use of a wireless- (rather than a wired) link for streaming audio and video introduces new challenges for the design of a home-networking system. The main challenge of a wireless entertainment system is to provide wire-equivalent quality in the presence of interference throughout the home environment in anywhere. Due to the isochronous character of streaming traffic accompanied with the substantial bandwidth need for video, the requirements that are imposed on the wireless channel are much higher than for traditional data applications. In ubiquitous home network, intelligent service begins in various information acquisitions. Sensor network is which can compose information network and the most suitable network to gather necessary information about users and environments. There are many wireless communication devices which support Ad-Hoc network topologies. The bluetooth is one of those systems provided network topology[1-3].

In this paper, to support the high data transmission rate, we propose a new method called 'DoublePico' in the Ad-Hoc network topology based on the bluetooth system.

In order to constitute the DoublePico, the node device which performs the functions of transmitted data relaying, has two bluetooth devices. The two bluetooth devices are run individually; one is run as a Master and other is run as a Slave. And, the node device forms two piconets via linking to the bluetooth devices on the other neighbor's node devices. Thereby, the Ad-Hoc network topology is created by diverse piconets which are formed two times.
Add more, the bluetooth devices of DoublePico use an ACL link which is defined in the bluetooth specification. The communication between two devices, which are constituted on their node, is performed by linking the wired data transmittable cable.

We show the results and the throughput which supports efficient data transmission rate, by running the simulator with Ad-Hoc network environments which are the proposed scenarios to get the simulation results. Then we show in this paper the efficiency of the DoublePico method through the analysis and the comparison from the existing algorithms of the bluetooth specification.

The rest of this paper is organized as follows. Section 2 shows related works, in section 3, to overcome the low data transmission rate, in the bluetooth Ad-Hoc network which is called scatternet and piconet in the bluetooth system, we present the DoublePico and a simulator. And, cope with a simulation model and SAW-ARQ scheme for the Bluetooth scatternet. In section 4, we get results by the simulation, and show the efficiency of the DoublePico in the final section.

\section{Related works}

The protocol presented in [8], BlueStars, proceeds from the device discovery phase into the following two phases of piconet formation and of the interconnection of the piconets into a connected scatternet. Based on a locally and dynamically computed weight (a number that expresses how suitable that node is for becoming a master) and on the knowledge of the weight of its neighbors (obtained during the discovery phase) each node decides whether it is going to be a master or a slave.

The main aim of the Yao protocol proposed in [7] is to build up a connected scatternet in which each piconet has no more than seven slaves.

The protocol assumes that each node knows its own identity, a dynamically computed weight that indicates how much that node is suitable for serving as a master (as in BlueStars), and its own location in the plane (usually provided by an on-board GPS device, or by any suitable inertial positioning system device). It is assumed that, as the outcome of the device discovery phase, a node also 
knows the identity of its neighbors, their weight and their location. By using the pecking protocol, the discovered devices also exchange information about their neighbors (achieving two-hop neighborhood knowledge).

\section{Wireless Home Networking using Bluetooth with SAW-ARQ}

We considered the way in which it supports the multimedia services based on WLAN and the way in which it gathers the environment information based on the sensor network.

In this paper, we form an Ad-Hoc environment by using bluetooth system technology which is one of the widely used technologies in the wireless devices. The data transmission rate is too low to transmit the amount of data packets such as multimedia data in the scatternet which is constructed by piconets to perform an Ad-Hoc network in the bluetooth network system. To support the high data transmission rate, we propose a new method called 'DoublePico' in the Ad-Hoc network topology based on the bluetooth system. One of the other two terminals connected to operate as a piconet, this protocol is not a routing algorithm.

The protocol presented in [8], BlueStars, is allows for the multi-hop transmission to generate the scatternet by connecting the piconet.

Also Yao protocol proposed in [7] is configuration for the more efficient scatternet, thereafter each of the piconet awares of the location of each other. But like this paper, it can not transmit efficiently by connecting some of the piconet.

This paper aimes at more efficiently data transmission using Bluetooth technology in the real world, and designs to be more suitable for real life.

\subsection{DoublePico}

The bluetooth uses FHSS (frequency Hopping Spread Spectrum) method to reduce the interference with other wireless devices. This method implements the hopping rule defined by the Master. The bluetooth has 79 channels in their frequency area. It uses one channel, which is selected to transmit data in a slot of time.
At the same time, the data transmission between each two pair of other connected devices is possible by using different channels, which are selected for each of the links in an overlapped area, where different piconets are being formed.

We propose the new method to form an Ad-Hoc network topology called 'DoublePico' (Double Piconet) to overcome the low data transmission rate of the existing network topologies of bluetooth.

The device node which performs the functions of a data relaying in an Ad-Hoc topology, is constructed by two bluetooth devices; one is run as Master and other is run as Slave. Thus node performs the functions of both Master and Slave at the same time.

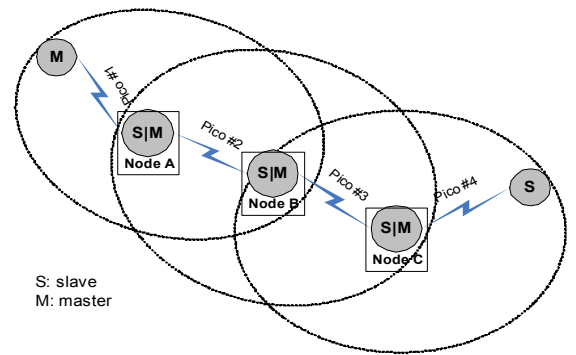

[Fig. 1] Ad-Hoc network by DoublePico method.

The nodes form piconets different from each other. By making a link to the bluetooth device of other node, the connection is formed between the device nodes. To form an Ad-Hoc network by the DoublePico method, the bluetooth devices on their node must not make a link to each other. Thus every node forms two different piconets, and finally forms an An-Hoc network topology, as shown in figure 1.

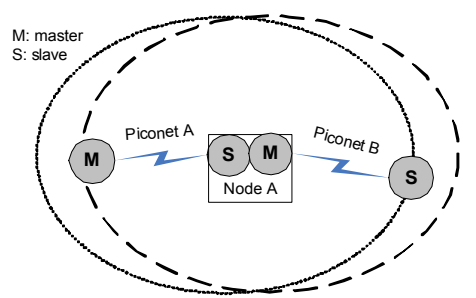

[Fig. 2] The gateway protocol stack in the embedded server

Because of the range of the radio frequency and two bluetooth devices at the same device node in the 
DoublePico method, there are two or more piconets on each node which performs a relaying of data packets to form an Ad-Hoc network topology, as shown in figure 2.

We can estimate the throughput of the DoublePico's method via the result values of the equations if they transmit a data packet via just one slot. The transmission of data in the DoublePico method has the advantage of supporting high data transmission rate, because the one of the bluetooths of nodes can have all slots to transmit data.

The data transmission between two bluetooth devices is possible in every slot area in a piconet (e.g. Piconet \#1) without interference with other piconets (e.g. Piconet \#2), because of FHSS method and the each bluetooth device independently runs in the piconets. Therefore, every bluetooth device always has got a chance for data transmission in the DoublePico.

When data transmissions are processing on each piconet (e.g. Piconet 1 and Piconet 2), the data transmissions are run individually on their piconet. In this case, the value of the probable number of data transmission successfully will be decreased, because the data transmission will be failed when the selected channels within the different piconets are the same either one or more time in the time duration of the slots, as A and $\mathrm{B}$ areas which are shown in the figure. Although the data transmission is transferred successfully in a slot before the 4 th slot range on the piconet 1 and the 12th slot range on the piconet 2 , both of data transmissions are failed by the same channels; the Slave 2 uses the 19th channel on the 5 th slot range on the piconet 1 , the Master 2 uses also the same channel on the 13th slot area on the piconet 2, and the time areas where the 5th area of slots on the piconet 1 and the 13th area of slots on the piconet 2 , are overlapped on the time axis.

The bluetooth system divides time equally and it is used by slot. It makes the collision when each piconet selects the same channels, but the data collision rate is a negligible quality during data transmission in a slot; it was shown by the mathematical equations and it will be shown in the results of simulation of the following sections. Therefore it supports the stability of transmitted data and makes data transmission rate constant.

\subsection{SAW-ARQ}

The process for sending sets of data on a uplink dedicated transport channel made of successive sub-frames from a mobile station to a base station of wireless communication network, said process including: implementing a SAW-ARQ process in which each set of data to be transmitted is split into packets which are transmitted in following sub-frames and a next packet is transmitted only when a response for the previous packet is received by the mobile station. It includes: defining several SAW channels, made of a sub-set of sub-frames regularly time spaced in the uplink dedicated transport channel; for each set of data, assigning a primary channel to said set of data, and sending the packets of said set of data in the sub-frames of said assigned primary channel by implementing a SAW-ARQ process in the primary channel.

In figure 3, unnumbered ARQ scheme is used to inform the source of the success of failure of transfer of payload. The baseband packets are retransmitted till a positive acknowledgement (ACK) is returned before timeout is exceeded. An optional 2/3 rate FEC (Forward Error Correction) can be used on the data payload to decrease the number of retransmissions. The packet header is always protected by a $1 / 3$ rate FEC since it contains valuable link information and should be able to sustain more errors.

On the ACL link, an ARQ scheme can be applied. In this scheme, a packet retransmission is carried out if the reception $\mathrm{CRC}$ (Cyclic redundancy check) to check for errors. Bluetooth has implemented a fast unnumbered ARQ scheme where the sender is notified of the packet reception in the RX slot directly following the TX slot in which the packet was sent [6].

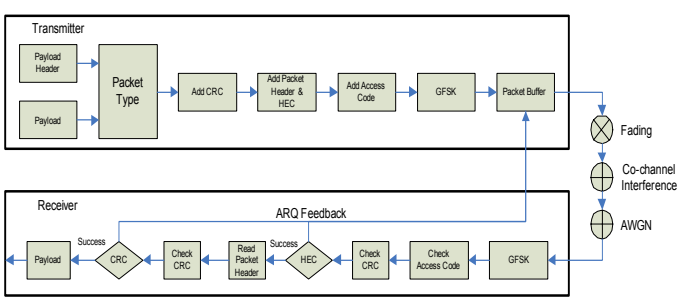

[Fig. 3] Block Diagram of DoublePico with SAW-ARQ.

\section{Simulation Results and Discussion}

The hopping rate is 1600 hops/sec and the bandwidth 
is $1 \mathrm{MHz}$. The gross bit rate is $1 \mathrm{Mbps}$ within a $1 \mathrm{MHz}$ channel bandwidth on a GFSK modulated carrier with $\mathrm{BT}=0.5$. Figure 4 depicts the simulation block diagram of the Bluetooth scatternet with ARQ scheme. In the block diagram, CRC and HEC coding are available. They are rate $2 / 3$ shortened Hamming code $(15,10)$ and rate $1 / 3$ bit repeat. An independent, static Rician fading channel was assumed for every packet. The algorithm is proposed to choose the suitable Bluetooth data packet for transmission through maximizing the expected throughput efficiency of ARQ protocol on Bluetooth ACL data communication link.

\subsection{The simulation of DoublePiuco}

The specification of the bluetooth systems defines many types of connection links for transmitting data packets in an ACL (Asynchronous Connection-Less) link between two devices. The DoublePico method, proposed in this paper, uses three types of data packet, which are shown in table 1 .

[Table 1] Data types in an ACL link.

\begin{tabular}{|c|c|c|c|c|}
\hline \multirow{2}{*}{ Type } & \multirow{2}{*}{ Slots } & \multirow{2}{*}{ Payload } & \multicolumn{2}{|c|}{ Asymmetric Max Rate } \\
\cline { 4 - 5 } & & & Forward & Reverse \\
\hline DH1 & 1 & 27 & 172.8 & 172.8 \\
\hline DH3 & 3 & 283 & 585.6 & 86.4 \\
\hline DH5 & 5 & 339 & 723.2 & 57.6 \\
\hline
\end{tabular}

If a bluetooth uses an ACL link with DH1 for data transmission between two devices connected, the duration of the slot timing is defined as $625 \mathrm{usec}$, and the data is transferred to connected device by one slot which is synchronized by a Master device. DH3 means that uses 3 slots to transfer data at one time, and DH5 uses 5 slots as well.

There are amount of header information in an ACL data packet and it takes a little time to wait for next slots which are allowed for sending a data packet by connected device's selves. Therefore, using three or more slots at one time is efficient to reduce total time of data transmission. The types of DH3 and DH5 are valuable when the bluetooth is needed for high data transmission rate.

In asymmetric transmission, when the bluetooth uses
DH5 packet type, the one side of devices uses 5 slots for sending a data packet, and the other side uses 1 slot to send. We ran the simulation applied to unidirectional data transmission between end devices. To support high rate, we used the asymmetric data transmission.

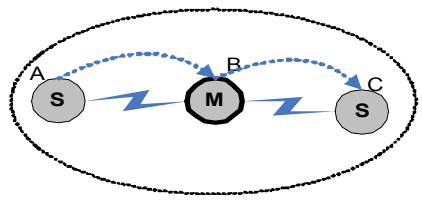

(a) in a piconet

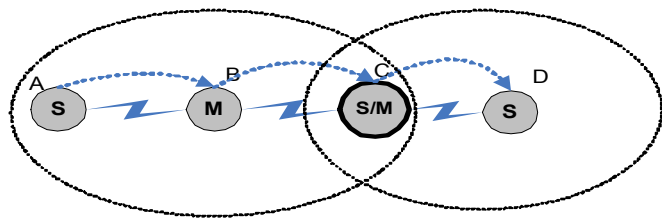

(b) in a scatternet

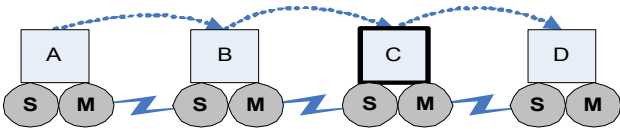

(c) in DoublePico method

[Fig. 4] Three scenarios for simulation.

To compare with other exist network topologies of the bluetooth system by the simulation, we made three scenarios, as shown in figure 4 . In the piconet environment such as (a), which is shown in the figure, for gathering the simulation results of the data transmission rate and the collision rate, we got the information from the node B which is running as Master. In this case, the data packets are sent from the node $\mathrm{A}$ to the node $\mathrm{C}$ through the node $\mathrm{B}$. The node $\mathrm{B}$ receives data packets from the node $\mathrm{A}$ and forwards data packets to the node C by using ACL link.

The data packets are flowed only to one direction. So, to use DH3 and DH5 for asymmetric data transmission, the node $\mathrm{B}$ assigned multi slots to node $\mathrm{A}$ for sending of the node A, uses one slot to reply for notifying an acknowledgement of node $\mathrm{A}$, and to forward to node $\mathrm{C}$, assigned one slot for receiving of reply about transferred data packets by using multi slots, as shown in figure 5 . Also the assignments of multi slots are the same in other 2 scenarios. The every slot synchronizations are managed by the node $\mathrm{B}$ in the only one piconet and there have to 
be no other piconets in neighborhood. Therefore there is no collision in the environment of this simulation.

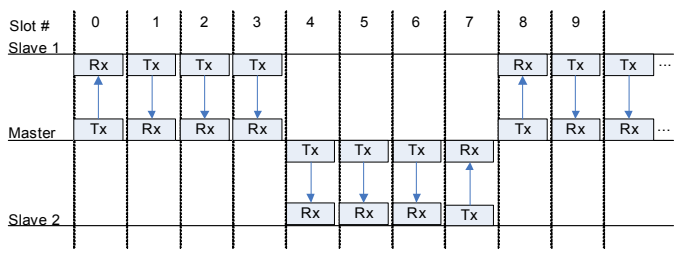

[Fig. 5] Forwarding data from Slave 1 to Slave 2 in a piconet.

In the case of the scatternet environment, as shown in the (b) of figure 8 , we got the results from the node $\mathrm{C}$ which is running as Master and Slave, but the node does not run as both (Master and Slave) at one time. This node is able to run as Slave for keeping the piconet connected with node B (e.g. left side) and receives data packets from the node B which is running as Master. The node $\mathrm{C}$ runs as Master to maintain a piconet which was formed before (e.g. right side) and forwards the received data packets to node D. Finally, the data packets are flowed from the node A to node D through the nodes B and C.

At last, we got the results from the node $\mathrm{C}$ in the DoublePico method which is proposed in this paper. The data packet is transferred from node A to node D. Each node has two bluetooth devices which run as Master and Slave, and two bluetooth devices on their node are able to communicate to each other by wired data cable.

For this simulation, we assumed that data delays and collisions were presented by overlapped slot timing and collisions at frequency channel. We didn't apply about the procedure of bluetooth connection rule within node devices and the creation of the route path. Moreover, to support highest transmission rate in bluetooth scatternet, we did not care about the process time for changing bluetooth's role: Master and Slave. So, we assume that all data is sent successfully if there are no collision and noise.

In this case of the piconet, there are no transmission errors for data transmission. Because the piconet is constructed by three bluetooth devices, and one is run as Master and the others are run as Slaves. The Master manages slot timing and the rule of the frequency sequences in their piconet, and there is just one piconet without any interference, so the slaves are operated only by those Master.

The BER of the DoublePico method is much higher than others, but the main focus of this paper is the high data transmission rate in the Ad-Hoc network topology based on the bluetooth system. Although of the highest BER, in the DoublePico method, there are many chances to transmit data packets.

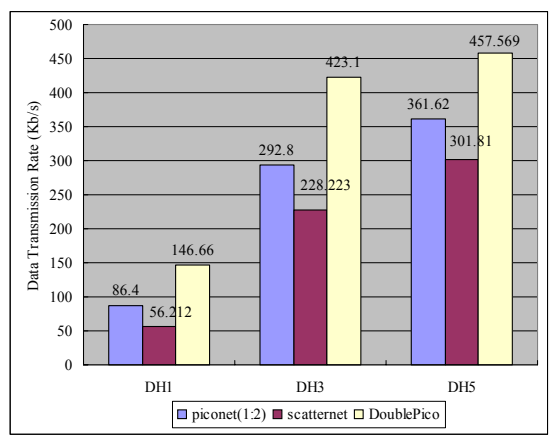

[Fig. 6] The simulation results.

As shown in figure 6, the data transmission rate is the highest when it is used by the DoublePico method and rate is the highest when the bluetooth uses DH5 packet type in ACL link.

The maximum data rate is $723.2 \mathrm{kbps}$ when it uses ACL link with DH5 packet type in a piconet which is constructed by two bluetooth devices. However, if there is only one Master and two Slaves in a piconet, the maximum data transmission rate is $361.6 \mathrm{kbps}$, because one Master has to support the same data rate for all connected Slaves in a piconet, or the devices must share the time. Moreover, there is the defect in a scatternet, what means the data transmission rate becomes low; the maximum rate is $302.2 \mathrm{kbps}$.

In this paper, we presented the method of DoublePico which supports about $457 \mathrm{kbps}$ of the maximum data transmission rate. Furthermore, to transmit the multimedia stream data, we used the mpeg4 encoder which is developed by MPEG4IP project[9][10]. The encoder supports many kinds of data types. Then we selected XviD codec and encoded the low multimedia data. And then, we could know the required data transmission rate to support real-time service stream, as shown in table 2 . 
[Table 2] Required transmission rate for XviD data service.

\begin{tabular}{|c|c|c|}
\hline Frame Size & frames/sec & kbps \\
\hline \multirow{3}{*}{$320 \times 240$} & 24 & 166.656 \\
\cline { 2 - 3 } & 32 & 222.208 \\
\cline { 2 - 3 } & 64 & 444.416 \\
\hline \multirow{3}{*}{$640 \times 480$} & 8 & 238.528 \\
\cline { 2 - 3 } & 12 & 357.792 \\
\cline { 2 - 3 } & 14 & 417.424 \\
\hline
\end{tabular}

As shown in the table, the data which is constructed of $320 \times 240$ is sufficient to transmit by DoublePico method, and we can see $640 \times 480$ data is possible to transfer not exceeding 14 frames/sec.

\subsection{The simulation of DoublePico with SAW-ARQ}

Figure 7 shows throughput improvement with SAW-ARQ scheme as a function of the number of active piconets. Using SAW-ARQ scheme, the throughput performances of the two packet types DH1, DM1 are significantly different. The length of the packet has a considerable affect on the throughput. The longer packets have the less chance of passing the CRC check as compared to shorter packets. Therefore, the throughput with small slots is greater than that with large slots for a given $\mathrm{Eb} / \mathrm{N} 0$ and number of piconet. As the number of retransmission increases from 0 to 10 , the throughput becomes more increased which consequently reduces the channel error. For DM1 packet, the number of retransmission, $\mathrm{L}=2$ provide for a maximal system capacity. For $\mathrm{DH} 1$ packet, $\mathrm{L}=9$ provide for a maximal system capacity. And a figure 8 shows the performance and BER with fading.

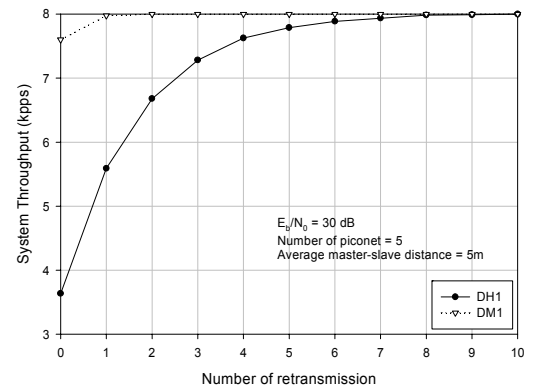

[Fig. 7] System Throughput Improvement of Bluetooth Scatternet using SAW-ARQ Scheme.

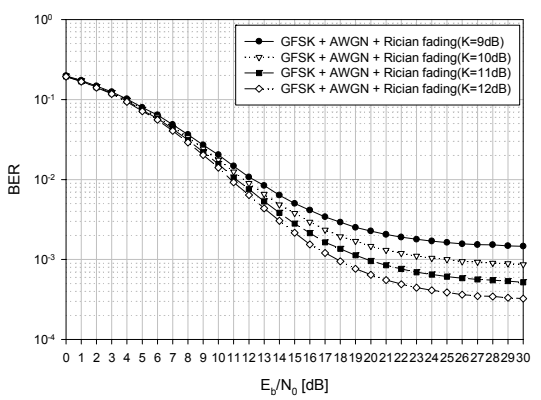

[Fig. 8] System Throughput Improvement of Bluetooth Scatternet using SAW-ARQ Scheme.

The performance evaluation is concentrated on the bit error rate (BER) to analyze feasibility of multimedia transmission. The wireless environment such as Bluetooth is characterized by limited bandwidth and high bit error rate. In the Bluetooth system SCO links are used for audio transmission, which is coded at a rate of $64 \mathrm{~Kb} / \mathrm{s}$. The real time video communication requests large bandwidth and delay restrictions. The MPEG-4 video compression standard is chosen to minimize the video transmission data rate. MPEG standard uses motion vectors between frames to encode temporal redundancy, and the discrete cosine transform to encode spatial redundancy. As we need large amount of compression, it introduces errors in the reconstructed video. The compressed video data is more sensitive to channel interference, because compression removes redundancy from the data. The MPEG-4 video compression standard incorporates several error tools to enable detection, containment, and concealment of errors. These techniques are successful in combating bit errors less than 10-3, but wireless channels can have much higher bit error rates. Especial difficult conditions of the mobile wireless channels result from rician fading due to motion between transmitter and receiver and changes in the surroundings. In our performance analyses we didn't assume any motion and mobility. The BER is important for QoS (Quality of Service) evaluation, because it strongly effects to radio link quality. In general, the theoretical value of BER can be computed if digital modulation scheme is specified. For shift keying, error probability is obtained as follows:

$$
P_{e}=\frac{1}{2} e r f c \sqrt{\frac{S}{N}}
$$


where $\mathrm{S}$ and $\mathrm{N}$ are signal and noise respectively, and erfc is the complementary error function, which is equal minus one the error function:

$$
\operatorname{erfc}(x)=1-\operatorname{erf} x=\frac{2}{\sqrt{\pi}} \int_{x}^{\infty} e^{-u^{2}} d u
$$

The noise disbibution is assumed to be Gaussian.

\section{Conclusion}

We have proposed the method called DoublePico to support high data transmission rate. This method has overcome the traditional low ad-hoc network topology.

Finally, if we take low cast into consideration, financial costs will be reduced to construct one node from two bluetooth devices. We think that method proposed in this paper can be referenced for many applications which need higher data transmission rate.

For gathering the good results of simulation, we have considered only one network topology with unidirectional path from the source to the destination device node, there is only one Master and one Slave in each piconet, so that data may be transmitted quickly without any interference in the simulation. As more devices are in a piconet, the wireless mesh routing mechanism are not considered. We leave this issue for future work.

\section{References}

[1] Telefonaktiebolaget LM Ericsson, International Business Machines Corporation, Intel Corporation, Nokia Corporation, Toshiba Corporation, "Specification of the Bluetooth system, Specification Volume 2, Core", Version 1.1b, February 2001.

[2] Telefonaktiebolaget LM Ericsson, International Business Machines Corporation, Intel Corporation, Nokia Corporation, Toshiba Corporation, "Specification of the Bluetooth system, Specification Volume 2, Profiles", Version 1.1b, February 2001.

[3] The Official Bluetooth Website, http://www.bluetooth.com

[4] Manthos Kazantzidis, "End-to-end Adaptive Multimedia over Bluetooth Scatternets", Proc. European Wireless 2002.

[5] J.Y.Khan and J.Wall, "Bluetooth-Based Wireless Personal Area Network for Multimedia
Communication", Proc. IEEE Computer Society 2002.

[6] Specification of the Bluetooth System, Core, Version 1.0B, December 1999.

[7] X. Li and I. Stojmenovic. Partial Delaunay triangulation and degree-limited localized Bluetooth scatternet formation. In Proceedings of AD-HOC NetwOrks and Wireless (ADHOCNOW), Fields Institute, Toronto, Canada, Sep. 2002.

[8] C. Petrioli, S. Basagni, and I. Chlamtac. Configuring BlueStars: Multihop scatternet formation for Bluetooth networks. IEEE Transactions on Computers, special issue on Wireless Internet (Y.-B. Lin and Y.-C. Tseng, eds.), 2002. in press.

[9] Chong Hooi Chia, M. Slim Beg, "Realizing MPEG-4 Video Transmission over Wireless Bluetooth Link via HCI", Proc. IEEE Transaction on Consumer Electronics, Vol.49, pp.1028-1034, Nov. 2003.

[10] The Website of MPEG4 Open Project Group, http://www.mpeg4ip.net

Sung-Hwa Hong

[Regular member]

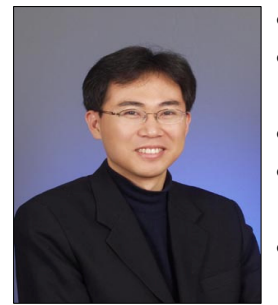

- Aug. 1996 : Korea Univ., BS

- Aug. 2002 : Korea Aerospace Univ., MS

- Aug. 2008 : Korea Univ., PhD

- Dec. $1997 \sim$ May. 2000 : Eastel system LtD., Engineer

- March. $2009 \sim$ current : Dongyang Technical College., Dept. of Software Eng.,, Lecturer

$<$ Research Interests $>$

Wireless Network, Ubiquitous, Home Network

Bong-Jik Kang

[Regular member]

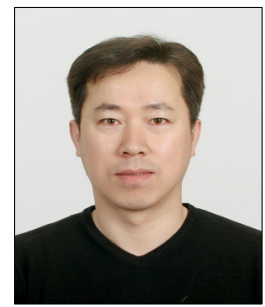

- Feb. 1989 : Seoul National Univ., BS

- Feb. 1991 : KAIST., MS

- Feb. 2004 : Ajou Univ., PhD

- 1991 1994 : Posdata LtD., Researcher

- Mar. $1995 \sim$ current : Dongyang Technical College., Dept. of Software Eng., Associate professor

$<$ Research Interests $>$

Information Communication, Information Management 